\title{
Gravitational waves perturbations of the early Universe
}

\section{Fomin I.V.}

Bauman Moscow State Technical University, Moscow, Russia;

E-mail: Fomin <ingvor@inbox.ru>;

The source of the anisotropy of the background radiation are the cosmological perturbations from a length of wave comparable or bigger Hubble radius. Such long-wave perturbations have come from an era when the Universe was much younger. The initial perturbations had the quantum mechanical nature and, subsequently, amplified an external gravitational field. Thus, the scalar and tensor perturbations are the source of information on the early Universe.

In this article the origin of the initial perturbations is considered and settled an invoice the power spectrum and the spectral indexes of the scalar and tensor perturbations within the exact solutions of the equations of dynamics of a scalar field.

Keywords: gravitational waves, scalar field, inflation.

DOI: $10.18698 / 2309-7604-2015-1-144-156$

\section{Introduction}

The cosmological perturbations are a source of the evolution of large-scale structure of the Universe. Generation of initial perturbations has the quantum-mechanical nature. The length of a wave of perturbations has strongly grown since generation, but other physical characteristics of the perturbations can still support the traces of their origin. The quantum mechanical generation of the cosmological perturbations depends only on existence their quantum fluctuations in the initial point and interaction of the perturbations with a variation gravitational field of the homogeneous isotropic Universe.

Gravitational waves are a valuable source of information about the stage of early evolution of the universe. State of accelerated expansion of the universe in the early stages describes the theory of inflation [1]. Inflationary cosmology explains the origin of the primary irregularities and predicts their range [2]. Thus, it is possible to test the theory by comparison with observational data.

According to the theory of inflation derived from primordial quantum fluctuations. These fluctuations have significant amplitude of the scale of the Planck length and for inflation they approached the scale of galaxies with almost the same amplitude. Thus, inflation connects the large-scale structure of the Universe with a microscopic scale. The resulting spectrum of inhomogeneities is essentially independent of particular scenarios of inflation and has a universal form. This leads to unambiguous predictions for the spectrum of the CMB anisotropy [2]. 
Inflation model are defined view of the effective potential $V(\phi)$. In this case, the potential is controlled by a scalar field $\phi$, which rolls down to the minimum $V(\phi)$. The end of inflation constitute a breach of the slow roll, field oscillates around the minimum and process of reheating begins. This process involves several different stages, such as the collapse of the inflation condensate (preheating), the production of particles of the standard model and thermalization [2].

The source of the CMB anisotropy are cosmological perturbations with wavelengths comparable to or greater than the Hubble radius. Initial perturbations have quantum-mechanical nature, and subsequently amplified by parametric external gravitational field. The wavelength of the perturbation has grown since the generation, but other physical characteristics of the perturbation may still bear traces of their origin. Quantum-mechanical generation of cosmological perturbations depends on the existence of quantum fluctuations in the inflationary stage and interaction of disturbances with variable gravitational field of a homogeneous isotropic universe.

Strong variable gravitational field of very early Universe plays the role of the pump field. It replaces the zero-point quantum perturbations and enhances them. The initial quantum state of each mode disturbances is transformed as a result of the quantum mechanical Schrödinger evolution to the "frozen" vacuum.

\section{Anisotropy of the microwave background}

The Universe just before recombination is a very tightly coupled fluid, due to the large electromagnetic Thomson cross section. Photons scatter off charged particles (protons and electrons), and carry energy, so they feel the gravitational potential associated with the perturbations imprinted in the metric during inflation. A density of baryons (protons and neutrons) does not collapse under the effect of gravity until it enters the causal Hubble radius. The perturbation continues to grow until radiation pressure opposes gravity and sets up acoustic oscillations in the plasma. Since densities of the same size will enter the Hubble radius at the same time, they will oscillate in phase. Moreover, since photons scatter off these baryons, the acoustic oscillations occur also in the photon field and induces a pattern of peaks in the temperature anisotropies in the sky, at different angular scales [3].

When the temperature drops and the expansion of the universe cools. Expansion rate is much slower characteristic time of establishment of equilibrium in hot plasma, so the particles in it are in thermodynamic equilibrium. One of these particles is relic photons.

Although photon propagates at the speed of light, in hot dense plasma due to scattering by electrons photons spread much more slowly. When the universe is expanding so that the plasma 
cools to a temperature of recombination, the electrons begin to connect with protons to form neutral hydrogen, and photons begin to spread freely.

Cosmic microwave background radiation has a unique property. Its temperature is remarkably isotropic. It is isotropic with precision. Nevertheless, there is a slight anisotropy. Anisotropy is due to the difference of temperature in different directions in the sky. Its magnitude is equal to about $3 \mathrm{mK}$. These kinetic components of the CMB anisotropy, which is called dipole anisotropy.

In addition to the kinetic component, there are potential members in the CMB anisotropy, owe their origin to the gravitational field of very large scale, which is comparable to the particle horizon, in other words, the distance to the last scattering neighborhood.

Consider the basic equation describing the anisotropy of the cosmic microwave background radiation, and the basic physical effects that it causes. The equation of change of temperature in the direction of $\mathbf{e}$ has the form

$$
\frac{\delta T(\mathbf{e})}{T}=-\frac{1}{2} \int_{\eta}^{\eta_{0}} d \eta \frac{\partial h_{i j}(\eta, \mathrm{r}(\eta))}{\partial \eta} e^{i} e^{j}+\frac{1}{4} \frac{\delta \rho}{\rho}+\left(\mathbf{e} \frac{\mathbf{v}}{c}\right)
$$

The first term in this equation describes the Sachs-Wolfe effect, which was predicted in the early 60s Sachs and Wolfe lies in the fact that photons moving in an alternating potential or gain or lose energy.

The second term is due to adiabatic preload radiation before the era of recombination in areas of high and low density Silk effect.

The third term owes its origin to the Doppler effect, which is a scattering of photons by moving adiabatic perturbations of free electrons before and after recombination.

The growing mode solution of the metric perturbation that left the Hubble scale during inflation contributes to the temperature anisotropies on large scales as

$$
\frac{\delta T}{T}(\theta, \varphi)=\sum_{I=2}^{\infty} \sum_{m=-l}^{l} a_{l m} Y_{I m}(\theta, \varphi)
$$

$a_{l m}$ - multipole coefficients, $Y_{l m}$ are the usual spherical harmonics. 
We can now compute the two-point correlation function or angular power spectrum $C(\theta)$ of the $\mathrm{CMB}$ anisotropies on large scales, defined as an expansion in multipole number

$$
C(\theta)=\left\langle\frac{\delta T^{*}}{T}(\mathbf{e}) \frac{\delta T}{T}\left(\mathbf{e}^{\prime}\right)\right\rangle_{\mathbf{e} \cdot \mathrm{e}^{\prime}=\cos \theta}=\frac{1}{4 \pi} \sum_{l=2}^{\infty}(2 I+1) C_{l} P_{l}(\cos \theta)
$$

where $P_{l}(\cos \theta)$ are the Legendre polynomials, and we have averaged over different universe realizations. Since the coefficients $a_{l m}$ are isotropic, we can compute the $C_{l}=\left\langle\left|a_{l m}\right|^{2}\right\rangle$ as

$$
C_{I}^{(s)}=\frac{4 \pi}{25} \int_{0}^{\infty} \frac{d k}{k} \mathcal{P}_{\mathcal{R}}(k) j_{I}^{2}\left(k \eta_{0}\right)
$$

where $j_{l}\left(k \eta_{0}\right)$ are spherical Bessel functions and $\eta_{0}$ is the distance to the surface of last scattering.

In the case of scalar metric perturbation produced during inflation, the scalar power spectrum at reentry is given by $\mathcal{P}_{\mathcal{R}}(k)=A_{S}^{2}\left(k \eta_{0}\right)^{n_{s}-1}$. In that case, one can integrate (7) to give

$$
C_{l}^{(s)}=\frac{2 \pi}{25} A_{s}^{2} \frac{\Gamma\left(\frac{3}{2}\right) \Gamma\left(I-\frac{n_{s}-1}{2}\right) \Gamma\left(I+\frac{n_{s}-1}{2}\right)}{\Gamma\left(\frac{3}{2}-\frac{n_{s}-1}{2}\right) \Gamma\left(I+2-\frac{n_{s}-1}{2}\right)}
$$

The tensor angular power spectrum can be expressed as

$$
\begin{gathered}
C_{l}^{(T)}=\frac{9 \pi}{4}(I-1)(I+1)(I+2) \int_{0}^{\infty} \frac{d k}{k} \mathcal{P}_{g}(k) I_{k l}^{2}, \\
I_{k l}^{2}=\int_{0}^{x_{0}} d x \frac{j_{2}\left(x_{0}-x\right) j_{1}(x)}{\left(x_{0}-x\right) x^{2}}
\end{gathered}
$$


where $x=k \eta$ and $\mathcal{P}_{g}$ is the primordial tensor spectrum.

The ratio of the tensor and scalar contribution to the angular power spectrum is $r=C_{l}^{(T)} / C_{l}^{(S)}$. Thus, the tensor angular power spectrum can be calculated as $C_{l}^{(T)}=r C_{l}^{(S)}$.

\section{Metric perturbations}

During inflation, quantum fluctuations of a scalar field will create metrics perturbations. Let's write down the metric in linear approximation, taking into account the scalar and tensor perturbations and the field perturbations [4]

$$
\begin{aligned}
& d s^{2}=a^{2}(\eta)\left[-(1+2 A) d \eta^{2}-2 B_{: i} d x^{i} d \eta+\left(\left(1+2 D_{i j}\right) \psi_{i j}+2 E_{: i j}+2 h_{i j}\right) d x^{i} d x^{j}\right] \\
& \phi=\phi(\eta)+\delta \phi\left(\eta, x^{i}\right)
\end{aligned}
$$

The functions of scalar perturbations $A\left(\eta, x^{i}\right), B, D, E$ depend on calibration, $\psi_{i j}$ is a metrics of space of constant curvature. The gauge-invariant tensor perturbations matches transverse and traceless gravitational waves $\nabla_{i} h_{i j}=h_{i}^{i}=0$.

In the article [5] the exact expressions for the power spectrum and the spectral indexes of the scalar and tensor perturbations were obtained.

$$
\begin{gathered}
\mathcal{P}_{\mathcal{R}}(k)=-\frac{H^{4}}{8 \pi^{2} \dot{H}}\left(\frac{k}{a H}\right)^{n_{S}-1}, \mathcal{P}_{T}(k)=\frac{H^{2}}{2 \pi^{2} M_{P}^{2}}\left(\frac{k}{a H}\right)^{n_{g}} \\
n_{s}-1=\frac{4 \dot{H}-\frac{H \ddot{H}}{\dot{H}+H^{2}}}{n_{g}}=\frac{H^{2}}{\dot{H}+H^{2}}
\end{gathered}
$$

where $H$ is the Hubble parameter, $M_{P}$ - Plank mass, $c=8 \pi G=8 \pi / M_{P}^{2}=1$.

\section{The basic equations}

The equations of a scalar field's dynamics in the flat Friedman-Robertson-Walker Universe are written as follows 


$$
\begin{aligned}
& 3 H^{2}=\left[\frac{1}{2} \dot{\phi}^{2}+V(\phi)\right] \\
& \ddot{\phi}+3 H \dot{\phi}+\frac{d V(\phi)}{d \phi}=0
\end{aligned}
$$

with the equation of state $p=w(t) \varepsilon$, where $p=\frac{1}{2} \dot{\phi}^{2}-V(\phi)$ is the pressure, $\varepsilon=\frac{1}{2} \dot{\phi}^{2}-V(\phi)$ energy density and $w(t)$ is the state parameter.

Because of the nonlinear character of Einstein's equations, exact solutions to the scalar cosmology equations can only be found using simple methods for very particular potentials. But it's difficult to find exact solutions for more appealing potentials. The slow-roll approximations is the usual procedure to receive the approximate solutions of these equations [5]

$$
\begin{aligned}
& 3 M_{P}^{2} H^{2} \approx V(\phi), \frac{1}{2} \dot{\phi}^{2} \rightarrow 0 \\
& 3 H \dot{\phi} \approx-\frac{d V(\phi)}{d \phi}=0, \ddot{\phi} \rightarrow 0
\end{aligned}
$$

A consequence of the system of equations $(5-6)$ can be written as

$$
3 H^{2}(\phi)-2\left(\frac{d H(\phi)}{d \phi}\right)^{2}=V(\phi)
$$

Then we write the state parameter $w(t)$ as follows 


$$
w(t)=\frac{\frac{1}{2} \dot{\phi}^{2}-V(\phi)}{\frac{1}{2} \dot{\phi}^{2}+V(\phi)}=1-\frac{2}{3} \frac{\dot{H}(t)}{H^{2}(t)}=1+\frac{4}{3}\left[\frac{d H(\phi) / d \phi}{H(\phi)}\right]^{2}=1-\frac{r(\phi)}{6}
$$

One can obtain from this equation

$$
8\left[\frac{d H(\phi)}{d \phi}\right]^{2}-r(\phi) H^{2}(\phi)=0
$$

The solution of the equation (8) allows getting the Hubble parameter, the potential and the scalar field from $r(\phi)$ :

$$
\begin{gathered}
H(\phi)=A \exp \left(\int \sqrt{r(\phi)} d \phi\right) \\
V(\phi)=A^{2}[3-8 r(\phi)] \exp \left(\int 2 \sqrt{r(\phi)} d \phi\right) \\
\dot{\phi}=-4 A \sqrt{r(\phi)} \exp \left(\int \sqrt{r(\phi)} d \phi\right)
\end{gathered}
$$

The exact solutions of the scalar field's dynamical equations can be obtained for different cosmological models by choosing of the $r(\phi)$. The power spectra and the spectral indices of scalar and tensor perturbations and the two-point correlation function of angular CMB anisotropies on large scales in the models of cosmological inflation can be calculated from $(2-5)$ on the basis of these exact solutions.

\section{The models of cosmological inflation}

Consider the constant tensor to scalar ratio $r(\phi)=B^{2}$ and obtain the solutions of $(9-11)$ 


$$
\begin{gathered}
V(\phi)=A^{2}(3-4 B) e^{ \pm B \phi} \\
\phi(t)= \pm \frac{1}{B} \ln \left[\frac{1}{2 A B^{2}(t+C)}\right] \\
H(t)=\frac{1}{2 B^{2}(t+C)} \\
a(t)=a_{0}(t+C)^{\frac{1}{2 B^{2}}}, C=\text { const }, \phi_{0}=0
\end{gathered}
$$

The resulting solutions correspond to the exponential inflation[6] For $r(\phi)=\frac{A^{2} \phi^{2}}{\left(A \phi^{2}+B\right)^{2}}$ we receive

$$
\begin{gathered}
V(\phi)=\frac{A^{2}}{3}\left(\phi^{2}-\frac{B}{A}\right)^{2} \\
\phi(t)=C \exp (-4 A t) \\
H(t)=A C^{2} \exp (-8 A t)+B \\
a(t)=a_{0} \exp \left[\frac{C^{2}}{8}\left(1-\exp \left(\frac{A t}{8}\right)\right)+B t\right]
\end{gathered}
$$

that corresponds to the model with spontaneous symmetry breaking[7] When $A=1, r(\phi)=\frac{\phi^{2}}{\left(\phi^{2}+B\right)^{2}}$ the solution of the system $(9-11)$ is defined as

$$
V(\phi)=3 C\left(\phi^{2}+B^{2}\right)\left(B^{4}+2 B^{2} \phi^{2}+\phi^{4}-6 \phi^{2}\right)
$$




$$
\begin{gathered}
\phi(t)=B \cosh (C t) \\
H(t)=C\left[B^{2} \cosh ^{2}(C t)+B^{2}\right]^{3 / 2} \\
a(t)=a_{0} \exp \left[\frac{A^{2}+\cosh ^{2}(C t)}{\cosh (C t)}\right]
\end{gathered}
$$

This is inflation with polynomial potential[8].

\section{The quintessential inflation}

Consider the tensor to scalar ratio as $r(\phi)=\tanh ^{2}(A \phi)$. The solution of the system $(9-11)$ gives the following expressions

$$
\begin{gathered}
V(\phi)=4 C^{2}\left(\frac{6}{B^{2}}+1\right)+C^{2}\left(\frac{12}{B^{2}}-2\right) e^{A \phi}+C^{2}\left(\frac{12}{B^{2}}-2\right) e^{-A \phi} \\
\phi(t)=\frac{1}{A} \ln \{\tanh [A B(t+C)]\} \\
H(t)=\frac{2 B}{A \sinh [2 A B(t+C)]} \\
a(t)=a_{0}\left\{\frac{\tanh ^{2}[B(t+C)]}{1-\tanh ^{2}[B(t+C)]}\right\}^{1 / A^{2}}
\end{gathered}
$$

This is the quintessential inflation[9]

$$
V(\phi)=V_{0}+F e^{A \phi}+G e^{-A \phi}
$$


with $V_{0}=4 C^{2}\left(\frac{6}{B^{2}}+1\right), F=G=C^{2}\left(\frac{12}{B^{2}}-2\right)$.

In case of $B=\sqrt{6}$ the potential is $V(\phi)=8 C^{2}=\Lambda$ which corresponds to the de Sitter solution [10].

The amplitude and spectral energy density parameter of the relic gravitational wave are defined as [11]

$$
\begin{gathered}
h_{G W}^{2}=\frac{H_{i n f}}{4 \pi^{2} M_{P}^{2}} \\
\Omega_{G W}=\frac{1}{\rho_{c}} \frac{d \rho_{G W}}{d \ln f}=\frac{|\beta|^{2} f^{4}}{\rho_{c}}, \rho_{c}=24 \pi H_{0},
\end{gathered}
$$

where $H_{\text {inf }}$ and $H_{0}$ are the Hubble parameters in the era of inflation, and in the modern era.

$$
\begin{aligned}
& \Omega_{G W}^{M D}(f)=\frac{3}{8 \pi^{2}} h_{G W}^{2} \Omega_{m 0}\left(\frac{f_{h}}{f}\right), f_{h} \leq f<f_{M D} \\
& \Omega_{G W}^{R D}(f)=\frac{1}{6 \pi} h_{G W}^{2} \Omega_{r 0}, f_{M D} \leq f_{h}<f_{R D} \\
& \Omega_{G W}^{k i n}(f)=\frac{3}{8 \pi^{2}} h_{G W}^{2} \Omega_{m 0}\left(\frac{f}{f_{R D}}\right), f_{R D} \leq f_{h}<f_{k i n},
\end{aligned}
$$

Where

$$
\begin{gathered}
f_{h}=\frac{1}{2} H_{0} \\
f_{M D}=\frac{3}{2 \pi} f_{h}\left(\frac{\Omega_{m 0}}{\Omega_{r 0}}\right)^{1 / 2}
\end{gathered}
$$




$$
\begin{aligned}
& f_{R D}=\frac{1}{4} f_{h}\left(\frac{\Omega_{r 0}}{\Omega_{m 0}}\right)^{1 / 2} \frac{T_{r h}}{T_{M D}} \\
& f_{k i n}=H_{k i n}\left(\frac{T_{0}}{T_{r h}}\right)\left(\frac{H_{r h}}{H_{k i n}}\right)^{1 / 3}
\end{aligned}
$$

where matter, radiation and kinetic energy dominated epochs are represented by "MD", "RD" and "kin" respectively. $\Omega_{m 0}$ and $\Omega_{r 0}$ are the present values matter and radiation energy density parameters respectively. reheating temperature and Hubble parameter are represented by $T_{r h}$ and $H_{r h}$ respectively and we have taken reheating temperature and Hubble parameter approximately same as the temperature and Hubble parameter at the end of inflation.

\section{The possibilities of experimental detection of gravitational waves}

One of the promising methods for increasing the sensitivity of gravitational antennas in high-frequency part of the spectrum is the use of low-frequency optical resonance (LOR) phenomenon, whose presence in the Fabry-Perot interferometer is set in the works[12]

The paper [13] shows that the minimum energy density of gravitational waves that can be detected by using a low-frequency resonance in the optical Fabry-Perot interferometer can be estimated by the formula

$$
\Omega_{G W}(f)>\sqrt{\frac{2 \pi \kappa}{c^{2} T \Delta}} \frac{8 \pi^{3} \hbar f^{9 / 3}}{3 H_{0}^{2} k W_{0}},
$$

where $\kappa$ - phase shift describing the setting of the interferometer $c$ - the speed of light, $T$ time averaging of the spectral density, $\Delta$ - loss per cycle reflections, $\hbar$ - Planck constant, $k$ - wave number, $W_{0}$ - the power of monochromatic laser light, $f$ - frequency of the gravitational waves. 


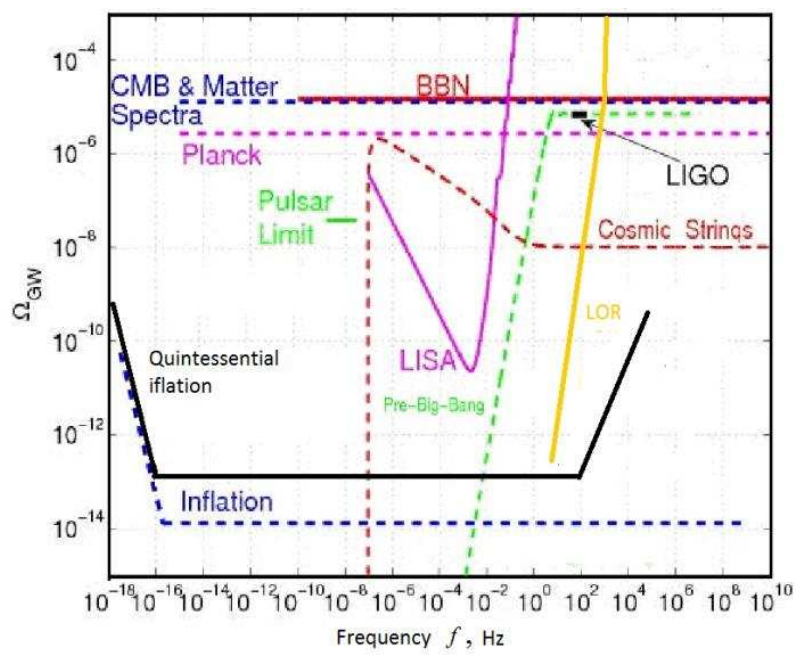

Fig. 1. The energy density of gravitational wave $\Omega_{G W}(f)$ for different models.

Figure 1 are graphs of energy density of gravitational wave for different models. The same graph shows the dependence of the minimum detectable energy density of gravitational waves for a variety of experiments and in the case of low-frequency optical resonance. It can be seen that at high frequencies in the model of quintessential inflation with the potential (12) for high frequencies $\Omega_{G W}$ increases.

The values of the frequency and the energy density of relic gravitational waves is limited by conditions[14]

$$
\int_{f_{0}}^{\infty} \Omega_{G W} d \ln f<1.1 \cdot 10^{-5}, f_{0} \approx 10^{-9} H z
$$

\section{Conclusion}

In this work we were derived exact solutions of scalar field's dynamical equations for the different models of inflation. Also presented the possibility of experimental detection of gravitational waves in the high frequency part of the spectrum using a low-frequency optical resonance phenomenon in the Fabry-Perot interferometer. The model parameters is limited by the influence of relic gravitational waves on the rate of primordial nucleosynthesis.

\section{References}

1. Liddle A.R., Parsons P., Barrow J.D. (1994). Formalizing the slow-roll approximation in inflation. Phys. Rev., D50, 7222-7232. 
2. Guth A.H., Kaisera D.I., Nomurab Y. (2014). Inflationary paradigm after Planck 2013. Phys. Lett., B, Vol. 733, 112-119.

3. Kotamatsu E., Smith K.M., Dunkley J., Bennett C.L., Gold B., Hinshaw G., Jarosik N., Larson D., Nolta M.R., Page L., Spergel D.N., Halpern M., Hill R.S., Kogut A., Limon M., Meyer S.S., Odegard N., Tucker G.S., Weiland J.L., Wollack E., Wright E.L. (2011). Seven-Year Wilkinson Microwave Anisotropy Probe(WMAP) Observations: Cosmological interpretation. Astrophys. J. Suppl., Vol. 192, 18.

4. Mukhanov V.F., Feldman H.A., Brandenberger R.H. (1992). Theory of cosmological perturbations. Phys. Rep., 215, 203-333.

5. Chervon S.V., Fomin I.V. (2008). About the calculation of cosmological parameters in exact models of inflation. Grav. \& Cosm., Vol.14, No.2, 163-167.

6. Copeland E.J., Liddle A.R., Wands D. (1998). Exponential potentials and cosmological scaling solutions. Phys. Rev., D57, 4686-4690.

7. Linde A.D. (1985). Chaotic inflation with constrained fields. Phys. Lett., B162, 281.

8. Bendyakov V.A., Giocaris N.D., Bendyakov A.V. (2008)ю On Higgs mass generation mechanism in the Standard Model. Phys. Part. Nucl., D39, 13-36.

9. Sa P.M., Henriques A.B. (2010). Gravitational-wave generation in hybrid quintessential inflationary models. Phys. Rep., D., V. 81, 124043.

10. Norbert Straumann (2006). From primordial quantum fluctuations to the anisotropies of the cosmic microwave background radiation. Ann. Phys, Leipzig, 15, No. 10-11, 701-845.

11. Sahni V., Sami M. (2004). Quintessential Inflation on the Brane and the Relic Gravity Wave Background. Phys. Rev., D70, 083513.

12. Gladyshev V.O., Morozov A.N. (1996). The theory of a Fabry-Perot interferometer in a gravitational wave experiment. J. Moscow Phys. Soc., V. 6, 209-221.

13. Esakov A.A., Morozov A.N., Tabalin S.E., Fomin I.V. (2015). Application of low-frequency optical resonance for registration of high-frequency gravitational waves. Vestnik MGTU.

Estestvennye nauki [Herald of BMSTU. Natural science], Vol. 1, 26-35.

14. Maggiore M. (2000). Gravitational Wave Experiments and Early Universe Cosmology. Phys. Rept., 331, 283-367. 